\title{
N,N-dimethyl Leucine Isobaric Labeling Reagent
}

National Cancer Institute

\section{Source}

National Cancer Institute. N,N-dimethyl Leucine Isobaric Labeling Reagent. NCI

Thesaurus. Code C161874.

A set of isobaric mass tags that contain dimethyl leucine. 